\title{
Correction to: Why People Forgive Their Intimate Partners' Infidelity: a Taxonomy of Reasons
}

\author{
Menelaos Apostolou $^{1} \cdot$ Andriana Demosthenous $^{1}$ \\ Published online: 19 November 2020 \\ (C) Springer Nature Switzerland AG 2020
}

\section{Correction to: Adaptive Human Behavior and Physiology https://doi.org/10.1007/s40750-020-00153-1}

The Online PDF version of the original article contained mistakes. The author noticed that in the HTML version, Tables 2 and 3 were presented correctly but in the Online PDF version, they are corrupted.

The original article has been corrected.

The online version of the original article can be found at https://doi.org/10.1007/s40750-020-00153-1

Menelaos Apostolou

m.apostolou@gmail.com

1 Department of Social Sciences, University of Nicosia, 46 Makedonitissas Ave, 1700 Nicosia, Cyprus 